\title{
Spin and Isospin of the Short Ranged Nucleon-Nucleon Interaction
}

\section{Igor Korover*}

Department of Physics, NRCN, P.O. Box 9001, Beer-Sheva 84190, Israel

igor.koroveregmail.com

The nuclear force that binds nucleons together is predominantly scalar, i.e., spin and isospin independent. The tensor, spin-isospin, and other components, are much weaker than the main scalar part. Since at typical nucleon distances in nuclei the scalar force is attractive, but becomes repulsive at shorter distances, it must vanish in between, and there the tensor force become important. The tensor force prefer the spins of the nucleons to be aligned (deuteron like configurations). Study of Short Range Correlated (SRC) nucleon pairs is a powerful way to investigate the short range tensor force and the even shorter range illusive repulsive force. Recent exclusive studies of SRC demonstrated that the single nucleon high - momentum distribution is not sensitive to nuclear structure and exhibits a universal scaling. The independence on the nuclear size enables the use of light nuclei for which experimental results can be compared to theoretical predictions. Data from a recent exclusive measurement on ${ }^{4} \mathrm{He}$ in Hall A, and from the data mining project of Hall B (CLAS), both in Jefferson Laboratory, VA, USA, is shown and discussed.

23rd International Spin Physics Symposium - SPIN2018 -

10-14 September, 2018

Ferrara, Italy

\footnotetext{
* Speaker.
} 


\section{SRC and Triple Coincidence Measurements}

The stability of atomic nuclei is the result of a delicate balance between a long-range attraction that binds the nucleons together and a short-range repulsion that prevent their collapse. Understanding the nature of these different parts of the nucleon-nucleon (NN) interaction and the resulting structure of nuclei and nuclear matter is a long-standing challenge of both theoretical and experimental nuclear physic. The NN interaction is dominated by scalar interaction thus, the NN potential is mainly spin and isospin independent. Normally the tensor part of the NN interaction is small compared to the dominant scalar part. It becomes important in the momentum range where the scalar force approaches zero, $0.8 \mathrm{fm}$. Measurements of Short Range Correlations (SRCs) in nuclei probe the tensor part of the NN force and even start to approach the repulsive part by studying the isospin decomposition of SRC pairs.

Nucleons in the nuclear ground state form nucleon pairs with large relative momentum and small center-of-mass $(\mathrm{CM})$ momentum, where large and small are relative to the Fermi momentum of the nucleus $\left(k_{F}\right)$. We refer to these pairs as short-range correlated (SRC) pairs. Recent highmomentum-transfer triple-coincidence studies [1-4] have shown that in the 300-600 MeV/c range of missing-momentum (the knocked-out proton's pre-scatter momentum in the absence of reinteractions), these pairs dominate the nuclear wave function, with neutron-proton (np) pairs nearly 20 times more prevalent than proton-proton ( $\mathrm{pp}$ ) pairs, and by inference neutron-neutron (nn) pairs. The strong preference for np pairs is due to the dominance of the tensor part of the NN interaction at the probed sub-fm distances [5-7].

The association of the small ${ }^{12} \mathrm{C}(\mathrm{e}, \mathrm{e}$ 'pp $) /{ }^{12} \mathrm{C}(\mathrm{e}, \mathrm{e}$ 'pn $)$ ratio, at missing momenta of 300-600 MeV/c, with dominance of the NN tensor force, leads naturally to the quest for increasing missing momenta. This allows the search for pairs at distances in which the nuclear force changes from being predominantly a tensor force to the essentially unexplored repulsive interaction. In a recent publication [4], a simultaneous measurement of the ${ }^{4} \mathrm{He}\left(\mathrm{e}, \mathrm{e}\right.$ 'p), ${ }^{4} \mathrm{He}(\mathrm{e}, \mathrm{e}$ 'pp $)$ and ${ }^{4} \mathrm{He}(\mathrm{e}, \mathrm{e}$ 'pn) reactions at (e,e'p) missing momenta from 400 to $830 \mathrm{MeV} / \mathrm{c}$ was reported. The measurements were motivated by the attempt to study the transition between the tensor-dominated regime to the short-range repulsive (and presumably scalar) NN force, using the isospin decomposition of $2 \mathrm{~N}-\mathrm{SRCs}$. The experiment was performed in Hall A of the Thomas Jefferson Laboratory (JLab) and the kinematics were chosen to cover a missing-momentum range of 400-830 MeV/c. For highly correlated pairs, the missing momentum of the $\mathrm{A}\left(\mathrm{e}, \mathrm{e}^{\prime} \mathrm{p}\right)$ reaction is expected to be balanced almost entirely by a single recoiling nucleon. The results of these measurements are shown in figure 1 (adapted from [4]).

\section{Universality of np-dominance}

Information on the dynamics of $2 \mathrm{~N}-\mathrm{SRC}$ pairs in heavier nuclei came from recent analysis of $\mathrm{A}\left(\mathrm{e}, \mathrm{e}^{\prime} \mathrm{p}\right)$ and $\mathrm{A}(\mathrm{e}, \mathrm{e}$ 'pp $)$ data on ${ }^{12} \mathrm{C},{ }^{27} \mathrm{Al},{ }^{56} \mathrm{Fe}$, and ${ }^{208} \mathrm{~Pb}$ targets, conducted as part of the Hall$\mathrm{B}$ data-mining project [8-10]. The analysis focused on large- $\mathrm{Q}^{2}, x_{B}>1.2$ kinematics, where the reaction is dominated by scattering off $2 \mathrm{~N}$-SRC pairs. In this kinematics, the $\mathrm{A}(\mathrm{e}, \mathrm{e}$ 'p) reaction is sensitive to both np- an pp-SRC pairs while the A(e,e'pp) reaction is sensitive to pp-SRC pairs. While this measurement did not probe directly np-SRC pairs, the fact that very few of the A(e,e'p) 


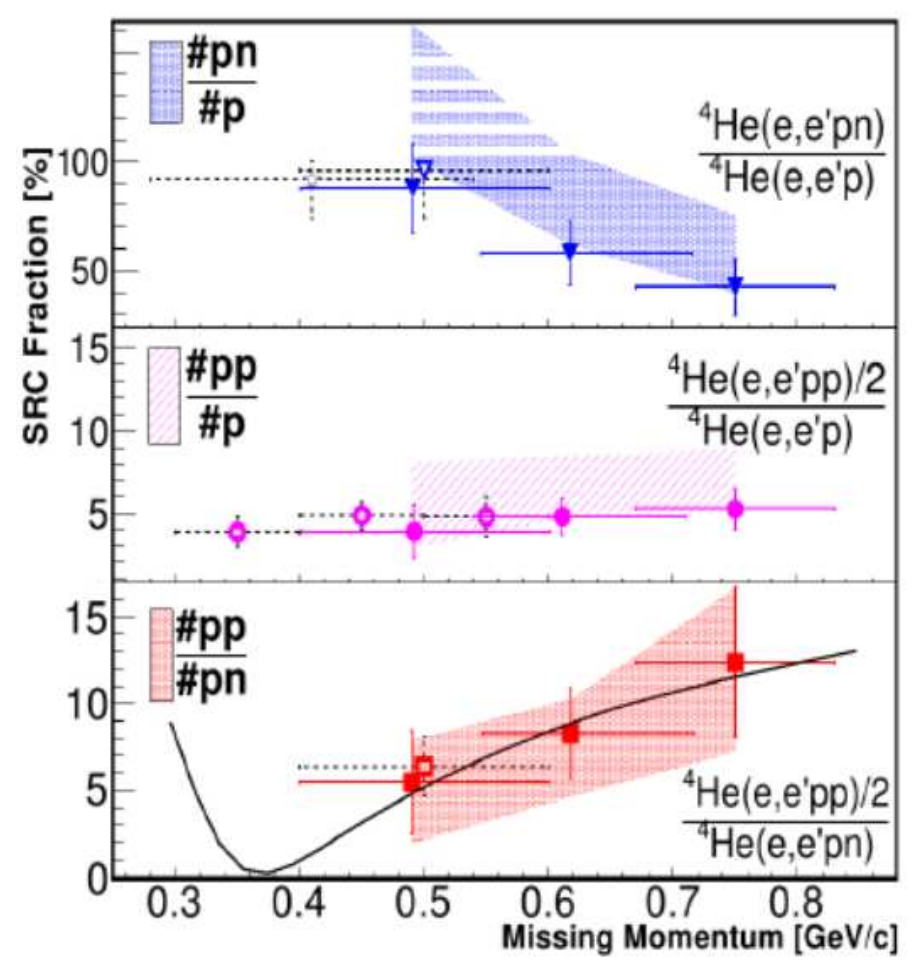

Figure 1: Lower panel: The measured ratio ${ }^{4} \mathrm{He}(\mathrm{e}, \mathrm{e}$ 'pp $) /{ }^{4} \mathrm{He}(\mathrm{e}, \mathrm{e}$ 'pn $)$ for ${ }^{4} \mathrm{He}(\mathrm{e}, \mathrm{e}$ 'p) missing momentum bins. Each point is the result for a different setting of the detectors. The bands represent the data corrected for Final State Interaction (FSI) to obtain the pair ratios. Solid black line shows VMC calculations, see [4] for details. The middle panel shows the measured ${ }^{4} \mathrm{He}(\mathrm{e}, \mathrm{e} ' \mathrm{pp}) /{ }^{4} \mathrm{He}\left(\mathrm{e}, \mathrm{e}^{\prime} \mathrm{p}\right)$, and extracted \#pp/\#p ratios. The upper panel shows the measured ${ }^{4} \mathrm{He}(\mathrm{e}, \mathrm{e}$ 'pn $) /{ }^{4} \mathrm{He}\left(\mathrm{e}, \mathrm{e}\right.$ 'p) and extracted \#pn/\#p ratios. Ratios for ${ }^{12} \mathrm{C}$ are shown as empty symbols with dashed bars. The empty star in the upper panel is the BNL result for ${ }^{12} \mathrm{C}(\mathrm{p}, 2 \mathrm{pn}) /{ }^{12} \mathrm{C}(\mathrm{p}, 2 \mathrm{p})$.

events were observed in coincidence with a correlated recoil proton, lead to the conclusion that there are very few pp pairs in the nucleus and the knocked out protons predominantly originated from np pairs. Figure 2 shows the fraction of $n p(p p)$ SRC pairs in the measured nuclei. As can be seen, np-SRC pairs are observed to dominate over pp-SRC pairs even in heavy, neutron rich, nuclei. The observed np-SRC dominance in heavy nuclei is a non-trivial result since in these heavy nuclei proton-proton pairs from different shells can create non $l=0$ pairs with non-zero spin, that are also sensitive to the tensor part of the $\mathrm{NN}$-interaction, thereby diminishing the observed np dominance in light nuclei. In the follow up analysis, where a direct measurement of the A(e,e'np) reaction was combined with A(e,e'pp) data, the ratio between pp to p SRC pairs was extracted, Figure 3.

\section{Residual A-2 system and Center of Mass motion of the SRC-pairs}

The use of light nucleus such as ${ }^{4} \mathrm{He}$, enabled the extraction of mass of the residual A-2 system. Following the ${ }^{4} \mathrm{He}(\mathrm{e}, \mathrm{e}$ 'pN) reaction, the residual system A-2 consist of two nucleons. As can be seen from figure 4, the missing mass is consistent with the assumption of 2N-SRC, where A-2 system is non interacting. Unlike the ${ }^{4} \mathrm{He}$, in Carbon and heavier nuclei the residual 


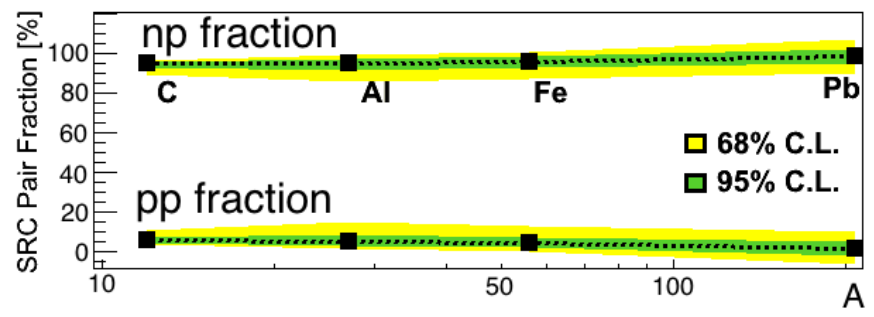

Figure 2: The extracted fractions of $n p$ (top) and pp (bottom) SRC pairs from the sum of pp and np pairs in nuclei in the missing-momentum range of 300-600 MeV/c. The green and yellow bands reflect $68 \%$ and $95 \%$ confidence levels, respectively [8].

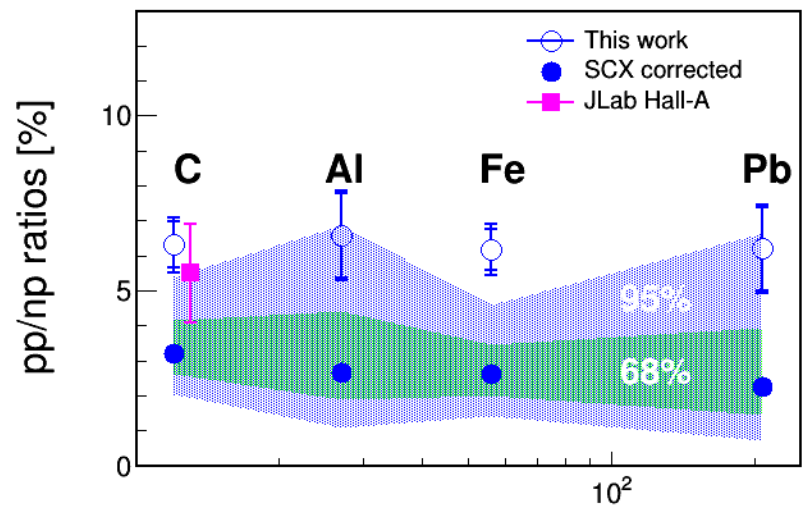

A

Figure 3: Extracted ratios of pp- to np-SRC pairs in nuclei [9].

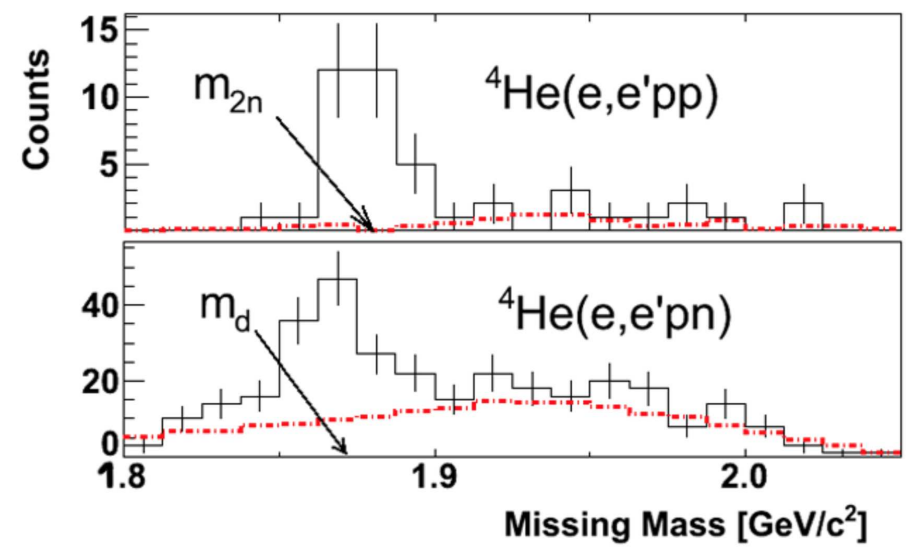

Figure 4: Missing mass distributions for the triple coincidence reaction [4]. 
system is complicated, thus missing mass is not a useful quantity to test if the A-2 system is at rest. However, measuring a small center of mass (CM) momentum of the pair compared to the large relative momentum between the nucleons is a fundamental characteristics of $2 \mathrm{~N}$-SRC pairs. It is an essential indications that the nucleons in the pair are in close proximity, with limited interaction with the surrounding nuclear environment, figure 5 [11].

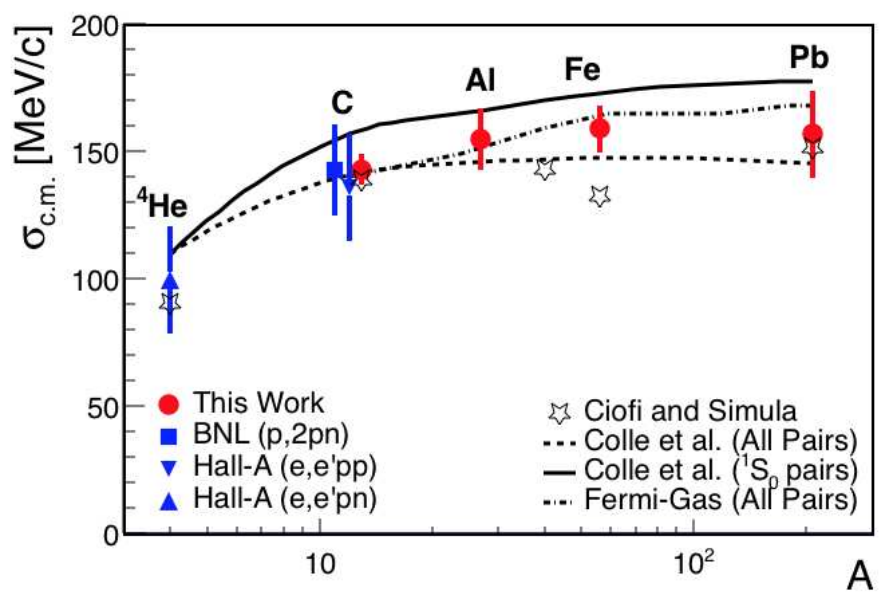

Figure 5: The nuclear mass dependence of the c.m. momentum distribution. The data points obtained in this work (red full circles) are compared to previous measurements (blue full squares and triangles) [3, 1 , 4] and theoretical calculations by Ciofi and Simula (open stars) [12], Colle et al., considering all mean field nucleon pairs (dashed line) and only 1S0 pairs (solid line) [13] and a Fermi-gas prediction [14] considering all possible nucleon pairs.

\section{Consequences of the np-dominance}

The observed dominance of the np-SRC pair implies that in heavy, neutron rich, nuclei the high-momentum tail contains the same amount of neutrons and protons, leaving the access neutrons to occupy low-momentum states (see insert in figure 6). Recent result shows that the fraction of high momentum protons increase as a function of neutron excess, while the relative amount of high momentum neutrons stays the same, see figure 6. This implies that in neutron rich nuclei there is an inversion of the momentum sharing between protons and neutrons where protons (i.e. the minority) have larger average momentum. This inversion is expected to be universal for any two-component Fermi systems with a short-range interaction between the different Fermions. This inversion and its universality has wide-ranging implications including prediction for Isospin dependence of the EMC effect, Figure 7 in neutron rich nuclei, the kinetic term of the nuclear symmetry energy at supra-nuclear densities, neutrino-nucleus scattering, and ultra-cold atomic gases.

\section{Summary}

Nucleon-Nucleon interaction is mainly scalar without spin dependence. However, in "limited" nucleon momentum range, where the Tensor part of the NN interaction is dominant, spin is important to the interaction. Existence of spin is important for np-dominance. The preference of the 


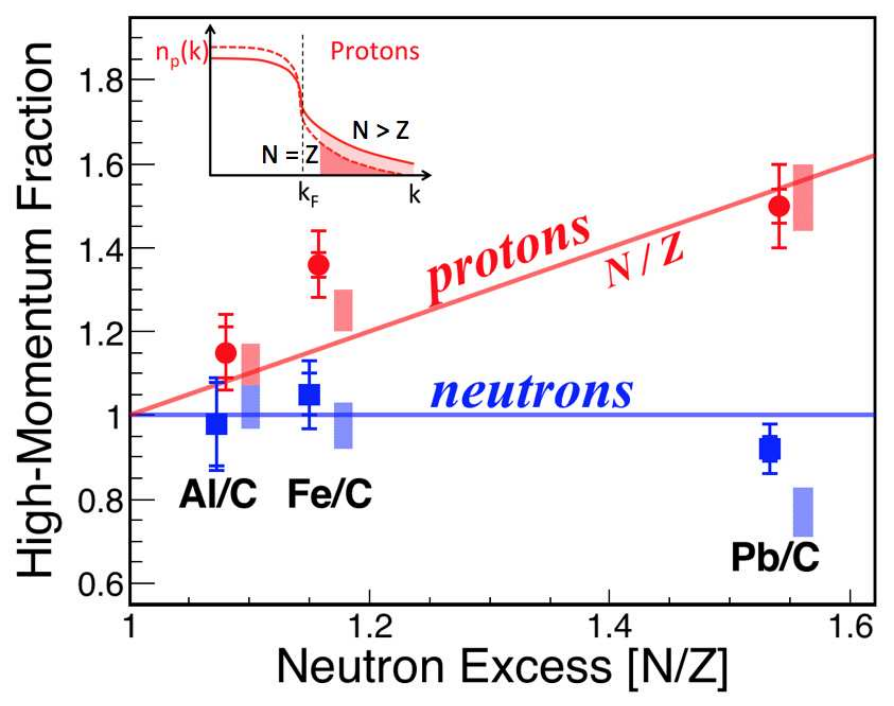

Figure 6: Relative high momentum fractions for neutrons and protons [9].

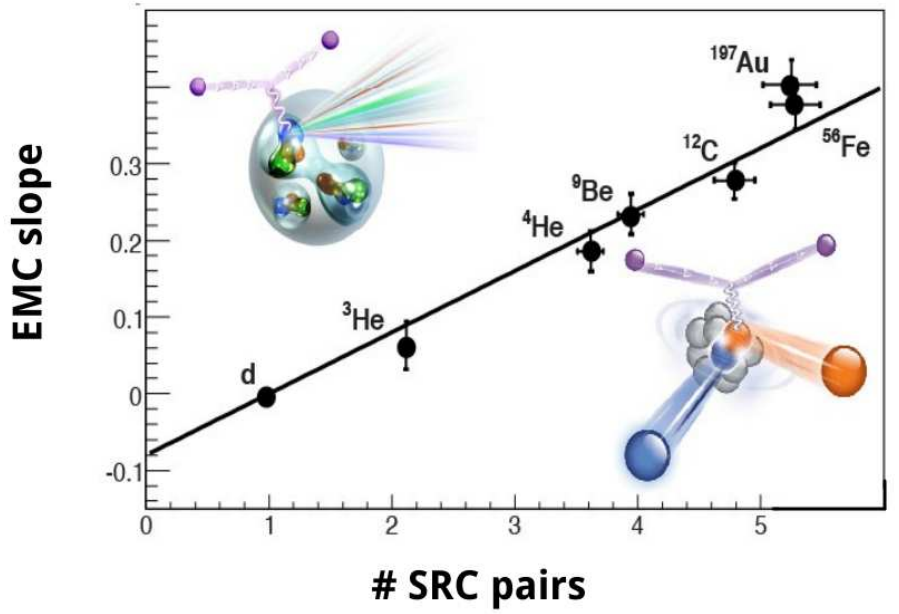

Figure 7: The strength of the EMC effect versus the corresponding number of SRC pairs.

$\mathrm{np}$-SRC pairs over all other pair types is also a dominance of $\mathrm{S}=1$ SRC pairs, and has a significant effect on nuclear properties, such as a possible source for nucleon modifications inside the nucleus and the kinetic energy distribution of nucleons in nuclei.

\section{References}

[1] R. Shneor et al., "Investigation of Proton-Proton Short-Range Correlations via the ${ }^{12}$ C(e,e'pp) Reaction.", Phys. Rev. Lett. 99, 072501 (2007).

[2] R. Subedi et al., "Probing Cold Dense Nuclear Matter." Science 320, 1476 (2008).

[3] A. Tang et al., "n-p Short-Range Correlations from $(p, 2 p+n)$ Measurements.", Phys. Rev. Lett. 90, 042301 (2003). 
[4] I. Korover et al., "Probing the Repulsive Core of the Nucleon-Nucleon Interaction via the ${ }^{4} H e(e, e$ 'pN) Triple-Coincidence Reaction.", Phys. Rev. Lett.113, 022501 (2014).

[5] R. Schiavilla, R. B. Wiringa, S. C. Pieper, and J. Carlson, "Tensor Forces and the Ground-State Structure of Nuclei.", Phys. Rev. Lett. 98, 132501 (2007).

[6] R. B. Wiringa, R. Schiavilla, S. C. Pieper and J. Carlson, "Dependence of two-nucleon momentum densities on total pair momentum.", Phys. Rev. C78, 021001 (2008).

[7] R. B. Wiringa, R. Schiavilla, S. Steven, C. Pieper, and J. Carlson, "Nucleon and nucleon-pair momentum distributions in A 12 nuclei.", Phys. Rev. C89, 024305 (2014).

[8] O. Hen et al., "Momentum sharing in imbalanced Fermi systems.", Science 346, 614-617 (2014).

[9] M. Duer et al., "Direct Observation of Proton-Neutron Short-Range Correlation Dominance in Heavy Nuclei.", arXiv:1810.05343 [nucl-ex] (2018).

[10] E. Cohen et al., "Center of Mass Motion of Short-Range Correlated Nucleon Pairs studied via the A(e,e’pp) Reaction.", Phys. Rev. Lett. 121, 092501 (2018).

[11] R. Weiss, R. Cruz-Torres, N. Barnea, E. Piasetzky, and O. Hen, "The nuclear contacts and short range correlations in nuclei.", Phys. Lett. B780, 211 (2018).

[12] C. Ciofi degli Atti and S. Simula, "Realistic model of the nucleon spectral function in few-and many-nucleon systems.", Phys. Rev. C53, 1689 (1996)

[13] C. Colle, W. Cosyn, J. Ryckebusch, and M. Vanhalst, "Factorization of exclusive electron-induced two-nucleon knockout.", Phys. Rev. C89, 024603 (2014).

[14] E. J. Moniz, I. Sick, R. R. Whitney, J. R. Ficenec, R. D. Kephart, and W. P. Trower, "Nuclear Fermi Momenta from Quasielastic Electron Scattering.", Phys. Rev. Lett. 26, 445 (1971) 